\title{
Can we trust small $x$ resummation?
}

\author{
Stefano Forte, ${ }^{\text {a }}$ Guido Altarelli, ${ }^{\mathrm{b}}$ Richard D. Ball ${ }^{\mathrm{c}}$ \\ ${ }^{a}$ Dipartimento di Fisica, Università di Milano and INFN, Sezione di Milano, \\ Via Celoria 16, I-20133 Milan, Italy \\ bDipartimento di Fisica "E.Amaldi", Università Roma Tre and INFN, Sezione di Roma Tre \\ Via della Vasca Navale 84, I-00146 Roma, Italy, \\ CERN, Department of Physics, Theory Division \\ CH-1211 Genève 23, Switzerland \\ 'School of Physics, University of Edinburgh \\ Edinburgh EH9 3JZ, Scotland
}

\begin{abstract}
We review the current status of small $x$ resummation of evolution of parton distributions and of deep-inelastic coefficient functions. We show that the resummed perturbative expansion is stable, robust upon different treatments of subleading terms, and that it matches smoothly to the unresummed perturbative expansions, with corrections which are of the same order as the typical NNLO ones in the HERA kinematic region. We discuss different approaches to small $x$ resummation: we show that the ambiguities in the resummation procedure are small, provided all parametrically enhanced terms are included in the resummation and properly matched.
\end{abstract}

\section{The need for small $x$ resummation}

The so-called small $x$ regime of QCD is the kinematical region in which hard scattering processes happen at a center-of-mass energy which is much larger than the characteristic hard scale of the process. An understanding of strong interactions in this region is therefore necessary to do physics at high-energy colliders. In this sense, HERA was the first small $x$ machine, and LHC is going to be even more of a small $x$ accelerator.

In deep-inelastic lepton-hadron interactions, the scale is set by the virtuality of the photon $Q^{2}$, and $x=\frac{Q^{2}}{2 p . q}=\frac{Q^{2}}{s}(1+O(x))$, where $s$ is the center-of-mass energy of the virtual photonhadron collision, and the distribution of partons which carry a fraction $x$ of the incoming nucleon energy is probed. In hadron-hadron interactions, the scale is set by the invariant mass $M^{2}$ of the final state, and $x=x_{1} x_{2}$, with $x_{i}=\frac{M}{\sqrt{s}} e^{ \pm y}, s$ the center-of-mass energy and $y$ the rapidity of the hadron-hadron collision. Here, the distribution of partons which carry fractions $x_{i}$ of the two incoming nucleon energies are probed. This means that typical $x$ values probed at the LHC in the central rapidity region are almost two orders of magnitude smaller than $x$ values probed at HERA at the same scale. Hence, small $x$ corrections start being relevant even for a final state with a characteristic electroweak scale $M \sim 100 \mathrm{GeV}$ (see Fig. 1).

As is by now well known, perturbative corrections become large at small $x$. Due to the accidental vanishing of some coefficients, the leading large corrections cannot be seen in NLO and NNLO splitting functions; however, the first subleading correction can already be seen in the NNLO splitting functions which have been computed recently, as well as in NNLO coefficient functions: they are large enough to make recent NNLO parton fits unstable at small $x$ (see Fig. 2).

This suggests dramatic effects from yet higher orders, so the success of NLO perturbation theory at HERA, as demonstrated by the scaling laws it predicts 34, has been for a long time very hard to explain. In the last several years this situation has been clarified, mostly thanks to the effort of two groups (ABF [5]-10] and CCSS [1]- 


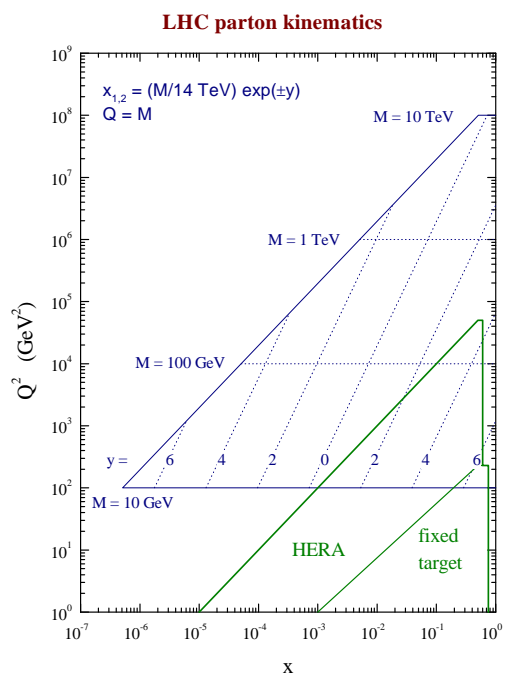

Figure 1. Comparison of the HERA and LHC kinematical regions (from Ref. [1]).

[16]), which have presented a full resummation of evolution equations in the gluon sector, thereby showing that, once all relevant large terms are included, the effect of the resummation of terms which are enhanced at small $x$ is perceptible but moderate - comparable in size to typical NNLO fixed order GLAP corrections in the HERA region. A detailed comparison of the $\mathrm{ABF}$ and CCSS approach in the pure gluon $\left(n_{f}=0\right)$ case was presented in Ref. [1, where excellent agreement was found.

This approach has now been generalized by $\mathrm{ABF}$ [17] to a full resummation including quarks, and including the resummation of deep-inelastic coefficient functions, so that resummed expressions for deep-inelastic structure functions can be obtained. Progress towards the inclusion of quarks has also been made by the CCSS group 18, though full results are not yet available. Meanwhile, an alternative somewhat simplified approach has also been developed [19], and used to perform a fit to deep-inelastic scattering data 20. In this approach, the factorization scheme is not defined in a fully consistent way at the resummed level, and also some contributions to the resummation are either not included, or treated by means of truncated expan-

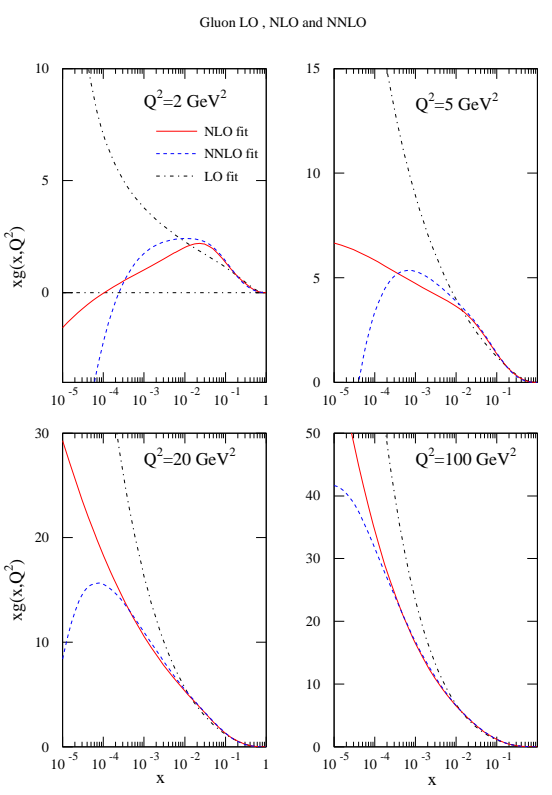

Figure 2. Comparison of the LO, NLO and NNLO gluon distributions in the MSTW08 parton fit (from Ref. [2]).

sions. A comparison of resummed predictions for deep-inelastic structure functions obtained in the TW and ABF schemes for resummation is presented in Ref. 21].

Here, we shall review the main ingredients that go into small $x$ resummation, with the aim of understanding the impact of various contribution on the final result and its stability upon higher order perturbative corrections and upon the inclusion of various subleading contributions: we shall take the $\mathrm{ABF}$ approach as a baseline, and discuss the impact of alternative options. We shall show that small $x$ resummation is very constrained by various requirements, which include momentum conservation, the inclusion of collinear contribution and matching to GLAP evolution, and consistency with the renormalization group. Once all these requirements are met, further subleading ambiguities are quite small; however, if some of the corresponding terms are missed out, effects are not negligible as we shall see. The resummed corrections thus obtained are perturbatively stable, as demonstrated by the fact that renormalization and factorization scale dependence are moderate, and decrease with increasing pertur- 
bative order as they ought to. The typical effect of the resummation in the HERA and LHC regions is comparable to that of NNLO corrections, but with the opposite sign.

In the next section, we shall review the ingredients which are necessary in order to perform the resummation in the gluon sector. In the subsequent section, we shall summarize the generic features of the resummed results. In the last section, we shall discuss how quarks and deepinelastic coefficient functions may be included in the resummation, and discuss resummed results for deep-inelastic physical observables.

\section{The three ingredients of stable resum- mation}

In this section, we discuss the resummation of evolution equations when $n_{f}=0$. In this case, there is a single parton distribution, the gluon distribution $G(\xi, t)$, with $\xi \equiv \ln \frac{1}{x}, t \equiv \ln \frac{Q^{2}}{\Lambda^{2}}$. It is convenient to define the Mellin transforms

$$
\begin{aligned}
G(N, t) & \equiv \int_{0}^{\infty} d \xi e^{-N \xi} G(\xi, t) \\
G(\xi, M) & \equiv \int_{-\infty}^{\infty} d t e^{-M t} G(\xi, t)
\end{aligned}
$$

\subsection{Double-leading expansion}

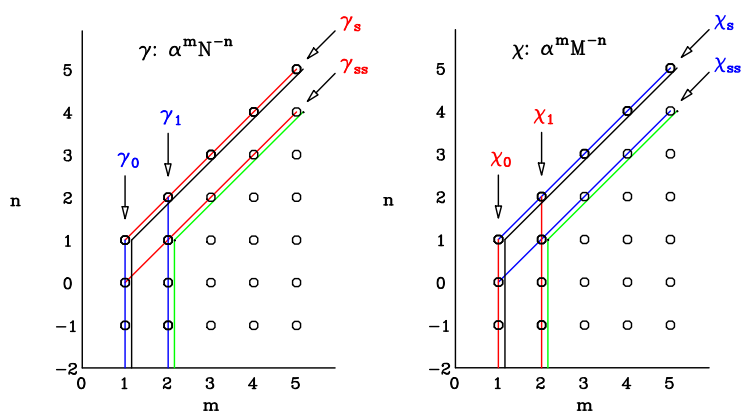

Figure 3. Double leading expansion of the GLAP anomalous dimension $\gamma$ (left) and the BFKL kernel $\chi$ (right).

The gluon distribution $G(N, t)$ can be expressed in terms of the gluon distribution at $t=t_{0}$ by solving the GLAP equation

$\frac{d}{d t} G(N, t)=\gamma\left(N, \alpha_{s}\right) G(N, t)$

which at the $\mathrm{N}^{k} \mathrm{LO}$ sums all terms of order $\alpha_{s}^{n} t^{n-k}$, to all orders in $\alpha_{s}$. The first step of resummation consists of including, to the $\mathrm{N}^{k} \mathrm{~L} \log$ level, all contributions to the anomalous dimension $\gamma\left(N, \alpha_{s}\right)$ of order $\alpha_{s}^{n} N^{-(n-k)}$, to all orders in $\alpha_{s}$, since they correspond to contributions of order $\alpha_{s}^{n} \ln ^{n-k} \frac{1}{x}$ to $G(\xi, t)$.

This inclusion is straightforward at the fixed coupling level, thanks to the fact that the gluon distribution $G(\xi, M)$ can be expressed in terms of the gluon distribution at $\xi=\xi_{0}$ by solving the BFKL equation

$\frac{d}{d \xi} G(\xi, M)=\chi\left(M, \alpha_{s}\right) G(\xi, M)$,

whose kernel $\chi\left(M, \alpha_{s}\right)$ is simply the inverse function of the GLAP anomalous dimension $\gamma\left(N, \alpha_{s}\right)$ 235]:

$\chi\left(\gamma\left(N, \alpha_{s}\right), \alpha_{s}\right)=N$.

The duality equation (4) maps the perturbative expansion of $\gamma$ in powers of $\alpha_{s}$ at fixed $N$ in the expansion of $\chi$ in powers of $\alpha_{s}$ at fixed $M$ and conversely. One can thus construct a double leading expansion [22] (see Fig. 3) which includes in $\chi$ all terms up to a given order in the expansion in powers of $\alpha_{s}$ both at fixed $M$ and at fixed $\frac{\alpha_{s}}{M}$. Its dual $\gamma$ can be shown to include terms up to the same order in the expansion in powers of $\alpha$ both at fixed $\alpha_{s}$ and at fixed $\frac{\alpha_{s}}{N}$.

Using either the double-leading $\chi$ or the double leading $\gamma$ in the BFKL or GLAP equation respectively leads to a solution which includes all terms which are logarithmically enhanced either in $\frac{1}{x}$ or in $Q^{2}$ to the given order [6]. The result (see Fig. (4) is close to the GLAP one when $M \rightarrow 0$, and close to the BFKL one when $N \rightarrow 0$. Because the perturbative expansion of the BFKL kernel is very poorly behaved, this resummed result has poor perturbative stability as $N \rightarrow 0$.

\subsection{Exchange symmetry}

The perturbative instability of the kernel as $N \rightarrow 0$ can be cured by observing that the BFKL 


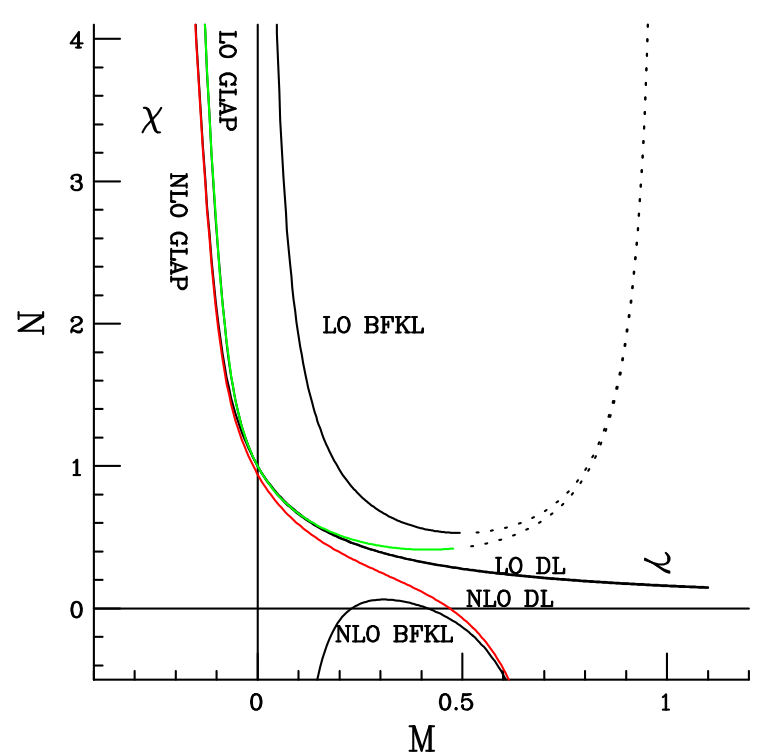

Figure 4. The BFKL kernel and its dual GLAP anomalous dimension computed at LO and NLO in the BFKL expansion, the GLAP expansion and the double-leading expansion.

kernel must be symmetric upon the interchange $M \rightarrow 1-M$, due to the symmetry of the threegluon vertex upon the interchange of the radiated and radiating gluon [13. This symmetry, which is manifest in the LO BFKL kernel, is however broken beyond the LO of the BFKL expansion by running coupling correction and by the choice of DIS kinematics [11]13. Nevertheless, the symmetry breaking terms can be computed exactly. Hence, one can symmetrize the double-leading expansion by undoing the symmetry breaking terms (by changing kinematics and argument of the running coupling), then symmetrizing the results, and finally restoring the original symmetrybreaking kinematics and choice of argument of $\alpha_{s}$ [10].

The result turns out to be surprisingly stable because of two features: (a) the anomalous dimension must satisfy the momentum conservation constraint $\gamma\left(1, \alpha_{s}\right)=0$, which by duality implies $\chi\left(0, \alpha_{s}\right)=1$; the anomalous dimension $\gamma\left(N, \alpha_{s}\right)$ decreases monotonically as $N$ increases as a consequence of the fact that a gluon looses momentum when radiating. Combining these two, it

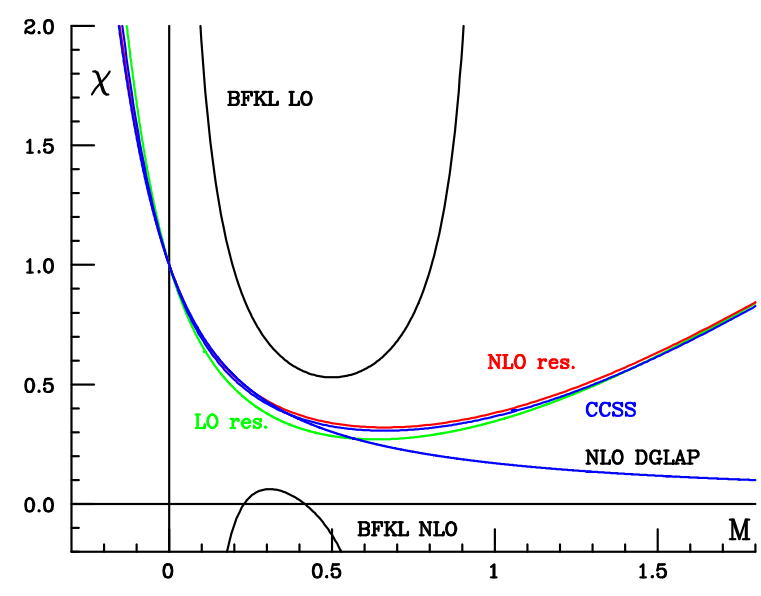

Figure 5. The LO and NLO resummed symmetrized double-leading kernels compared to the $\mathrm{LO}$ and NLO kernels in the BFKL expansion and the NLO GLAP kernel. CCSS denotes the corresponding result of Ref. [15] (from Ref. [1])

follows that $\chi$ always has a minimum, because it must go through the value $\chi=1$ at a pair of values of $M$ which are symmetric about the minimum, located at $M=\frac{1}{2}$. The minimum is preserved even when the symmetry is broken, in which case the two "momentum conservation" points at which $\chi=1$ get shifted to $M=0$ and $M=2$. One can further show that when the symmetry breaking is removed, the kernel is an entire function in the $M$ plane; after restoring the symmetry breaking it remains free of singularities for $\operatorname{Re} M>-1$ [10.

The perturbative instability of the BFKL kernel is thus completely removed: see Fig. 5. In this Figure we also compare results with those obtained by CCSS through a procedure which is rather different, but shares the following features: (a) all logarithmically enhanced terms in $Q^{2}$ and $\frac{1}{x}$ are included up to NLO (b) the underlying symmetry is implemented up to subleading terms. The extreme similarity of the results demonstrates the stability of the procedure.

\subsection{Running coupling}

The double-leading expansion upon which the resummation has been based so far sums up all terms which are large when $\alpha_{s}$ is small, but $\alpha_{s} \xi=\alpha_{s} \ln \frac{1}{x} \sim 1$ : namely, a contribution of the 
form $F\left(\alpha_{s} \xi\right)$ to the splitting function is considered to be of order $\alpha_{s}^{0}$. Thus, if the computation is performed at $\mathrm{N}^{k} \mathrm{~L}$ order, relative corrections are of order $\alpha_{s}^{k+1}$ when $\alpha_{s} \rightarrow 0$ while either $x$ or $\alpha_{s} \xi$ are kept fixed. However, this does not guarantee that corrections remain small if $\xi \rightarrow \infty$ (i.e. $x \rightarrow 0$ ) with $\alpha_{s}$ fixed. It is easy to see [5] that any correction which changes the leading (rightmost in the $N$ plane) small $N$ singularity of the anomalous dimension leads to a contribution to the splitting function which blows up as $\xi \rightarrow \infty$ in comparison to the lower order, because such a contribution changes the asymptotic $x \rightarrow 0$ behaviour of the splitting function, and thus of the parton distribution.

The leading singularity of the anomalous dimension is a simple pole at $N=0$ in the LO (and NLO) GLAP case. After double leading resummation it becomes a square-root branch cut: the inverse dual Eq. (4) of the quadratic behaviour of the kernel near its minimum. As discussed in Sect. 2.2 the presence of a minimum of the kernel is a generic feature which follows from its symmetry properties and momentum conservation. The intercept of the kernel however changes order by order, and it is only its all-order position which determines the asymptotic small $x$ behaviour. Hence, higher order corrections to the position of the minimum are asymptotically large as $\xi \rightarrow \infty$ : this suggests that the all-order location of the minimum of the kernel should be treated as a non-perturbative parameter, to be fitted to the data 677.

However, running coupling corrections change this state of affairs. Because the running of the coupling is subleading in $\ln \frac{1}{x}$, running coupling corrections only affect the duality equation (4) through a finite number of terms: at the $\mathrm{N}^{k} \mathrm{~L}$ logarithmic level, the duality relation is corrected by a term of the form

$\gamma_{\beta_{0}}^{(k)}=\left(\alpha_{s} \beta_{0}\right)^{k} f_{\beta_{0}}^{(k)}\left(\frac{\alpha_{s}}{N}\right)$,

where the function $f_{\beta_{0}}^{(k)}\left(\frac{\alpha_{s}}{N}\right)$ can be calculated at any given order using suitable operator methods in terms of the $\mathrm{N}^{k} \mathrm{LO}$ BFKL kernel [24].

Now, it turns out that whenever the fixed coupling kernel has a minimum, the running cou- pling correction $\gamma_{\beta_{0}}^{(k)}$ is also asymptotically large as $\xi \rightarrow \infty$ : in fact $f_{\beta_{0}}^{(k)}$ Eq. (5) grows as $\alpha_{s}^{n} \xi^{n}$ in comparison to the splitting function computed at LO in the double-leading expansion. Hence, in order to determine the correct $\xi \rightarrow \infty$ limit we must resum all these terms. This resummation can be performed exactly for the series of terms which grow fastest as $\xi \rightarrow \infty$ [8]: the result can be expressed in terms of Airy functions for a kernel which is linear in $\alpha_{s}$, and Bateman functions for a kernel with a generic dependence of $\alpha_{s}$ [10]. The (asymptotic, divergent) expansion of these functions in powers of $\alpha_{s}$ at fixed $\frac{\alpha_{s}}{N}$ gives back, to each order in $\alpha_{s}$, the contribution which upon inverse Mellin transform grows fastest as $\xi \rightarrow \infty$ to the terms $\gamma_{\beta_{0}}^{(k)}$ Eq. (5).

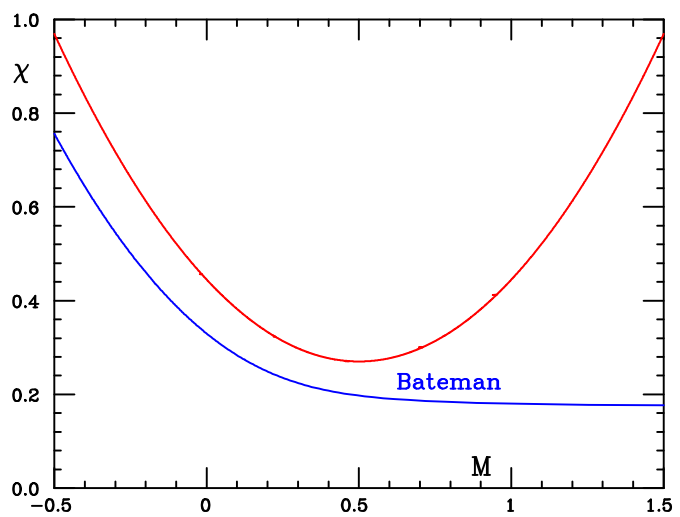

Figure 6. Quadratic approximation to the NLO resummed kernel of Fig. [5 and its Bateman running-coupling resummation.

It can then be seen that the resummation of running coupling corrections changes the nature of the leading singularity: the fixed-coupling square-root branch cut is turned back into a simple pole. This is shown in Fig. [6] where we display the quadratic approximation to the doubleleading NLO kernel of Fig. [5, and its Bateman resummation, i.e. the anomalous dimension which is obtained from it when running coupling corrections to Eq. (5) it are included to all orders (computed using the dependence on $\alpha_{s}$ of the NLO resummed result of Fig. 河). 
Hence, after running coupling resummation, the minimum of the kernel no longer provides the leading small $x$ singularity, which is instead given by the pole in the Bateman anomalous dimension. The location and residue of the Bateman pole are fully determined by the intercept and curvature of the minimum of the original kernel, and their dependence on $\alpha_{s}$.

\section{General features of resummed results}
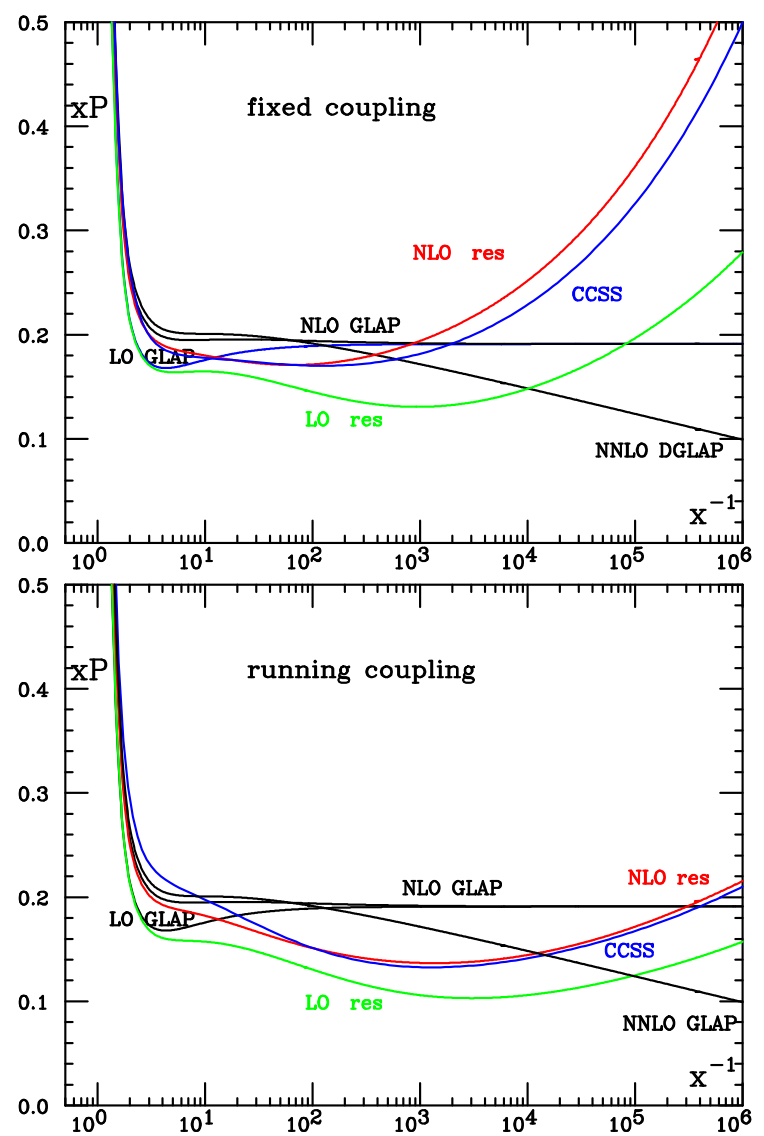

Figure 7. The resummed splitting function with $n_{f}=0$ and $\alpha_{s}=0.12$ with fixed coupling (top) and running coupling (bottom).

The crucial feature of the resummation procedure summarized in the previous section is that at each step the contributions which are included in the anomalous dimension on top of its standard GLAP fixed-order expansion in powers of $\alpha_{s}$ at fixed $N$ can be expanded out perturbatively, so that it is possible to obtain a fully resummed expression by simply combining all contributions, and subtracting the double counting terms. The procedure can be performed order by order in perturbation theory, by starting with the double-leading expansion of Fig. 3 to any given order, and then improving it as discussed in the previous section.

The resummed anomalous dimension has then schematically the form

$$
\begin{aligned}
& \gamma_{\Sigma N L O}^{r c}\left(\alpha_{s}(t), N\right)=\gamma_{\Sigma N L O}^{r c, \text { pert }}\left(\alpha_{s}(t), N\right) \\
& +\gamma^{B}\left(\alpha_{s}(t), N\right)-\gamma_{s}^{B}\left(\alpha_{s}(t), N\right) \\
& -\gamma_{s s}^{B}\left(\alpha_{s}(t), N\right)-\gamma_{s s, 0}^{B}\left(\alpha_{s}(t), N\right) \\
& +\gamma_{\text {match }}\left(\alpha_{s}(t), N\right)+\gamma_{\text {mom }}\left(\alpha_{s}(t), N\right),
\end{aligned}
$$

where

- $\gamma_{\Sigma N L O}^{r c, p e r t}\left(\alpha_{s}(t), N\right)$ is the fixed-coupling resummed anomalous dimension displayed in Fig. 5, obtained by duality Eq. (4) from the kernel which in turn is found by symmetrization (Sect. 2.2) of the NLO doubleleading kernel (Figs. 314);

- $\gamma^{B}\left(\alpha_{s}(t), N\right)$ is the Bateman anomalous dimension Fig. 6.

- $\gamma_{s}^{B}\left(\alpha_{s}(t), N\right), \gamma_{s s}^{B}\left(\alpha_{s}(t), N\right) \gamma_{s s, 0}^{B}\left(\alpha_{s}(t), N\right)$ are double counting subtractions between the previous two, namely the contributions to the LO and NLO terms $\gamma_{\beta_{0}}^{(k)}$ Eq. (5) which grow fastest as $\xi \rightarrow \infty$;

- $\gamma_{\text {mom }}$ subtracts subleading terms which would otherwise spoil momentum conservation;

- $\gamma_{\text {match }}$ subtracts any contribution which deviates from NLO GLAP and at large $N$ (which corresponds to large $x$ ) doesn't drop at least as $\frac{1}{N}$.

Note that the last two contributions are formally subleading: they are included in order to improve the matching to the GLAP anomalous dimension, in that they remove from the resumma- 
tion subleading contributions which may be nonnegligible in the large $x$ region where the resummation is not supposed to have any effect.

In Figure 7 we display the splitting functions obtained from Mellin inversion of the resummed anomalous dimension Eq. (7) at the fixed coupling level and at the running coupling level i.e. respectively without and with the inclusion of the Bateman contribution $\gamma^{B}\left(\alpha_{s}(t), N\right)$ and its associate double counting subtractions. The result is also compared to the CCSS result Ref. [15] (from Ref. 11). The resummed expansion is seen to be stable (the LO and NLO results are close), all the more so at the running coupling level. The resummed result matches smoothly to the GLAP result in the large $x \gtrsim 0.1$ region, but at small $x$ it is free of the instability which the GLAP expansion shows already at NNLO.

The comparison between results obtained with the $\mathrm{ABF}$ method discussed here and those by CCSS [15] is illuminating in various respects. The CCSS approach also includes the three ingredients discussed in the previous section - doubleleading resummation, symmetrization of the kernel, and running coupling corrections - but the implementation is rather different. In particular, the resummation of running coupling corrections is obtained by numerical solution of the runningcoupling BFKL equation (3) (see Ref. 1]). The closeness of results obtained by CCSS and ABF at the fixed coupling level reflects the closeness of the kernels Fig. 5. The fact that CCSS and $\mathrm{ABF}$ results are even closer at the running coupling levels follows from the softening of resummation effects due to asymptotic freedom. Also, the fact that exact numerical resolution of the running-coupling BFKL equation (in the CCSS approach) followed by a numerical extraction of the associate anomalous dimension, and the resummation of the leading running coupling corrections Eq. (5) in terms of a Bateman function (in the $\mathrm{ABF}$ approach) lead to a result which manifestly coincides for all $x \lesssim 0.03$ supports the accuracy of both procedures.

It is interesting to observe however that the CCSS resummed result shows a significant deviation from the unresummed GLAP result even for $x \gtrsim 0.1$, which is not seen in the ABF re- sult. Because this effect is only present at the running coupling level, it is likely to be due to the fact that running coupling terms lead to contributions which survive in the large $x$ region, where these terms are formally subleading (recall that the running coupling contributions were selected on the basis of their behaviour as $\xi \rightarrow \infty$ i.e. $x \rightarrow 0$ ). In the ABF approach (but not in the CCSS approach) these contributions are subtracted through the term $\gamma_{\text {match }}\left(\alpha_{s}(t), N\right)$ in Eq. (7). We have found this subtraction to be necessary in order for the resummed results not to differ from the fixed-order ones in the $x \gtrsim 0.1$ region.
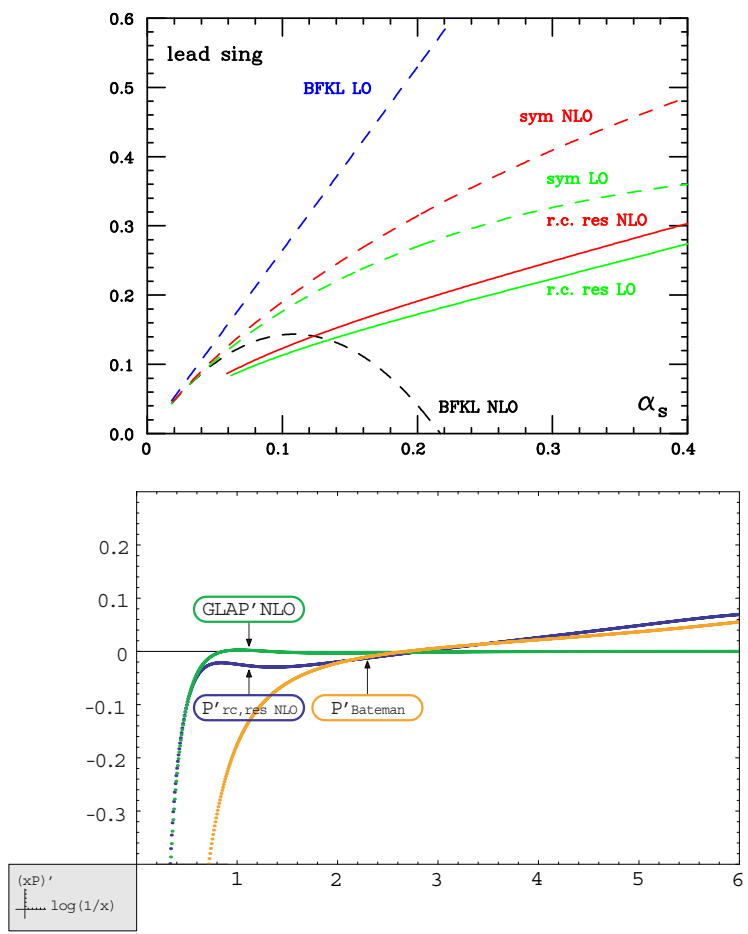

Figure 8 . The leading small $x$ singularity vs. $\alpha_{s}$ (top) and the slope of the splitting function vs. $\xi=\ln \frac{1}{x}$ (bottom, from Ref. [25]).

More detailed insight into the generic features of the resummation can be obtained by studying its small $x$ behaviour. As already mentioned, this is largely determined by the position of the leading (rightmost) singularity of the anomalous 
dimension in the $N$ plane. For the LO and NLO GLAP anomalous dimension this is located at $N=0$, while at the resummed level it is plotted as a function of $\alpha_{s}$ in Fig. 8 the large and perturbatively unstable corrections at the BFKL level (compare Fig. 4) are turned into more moderate and stable correction (compare Fig. 5) by the symmetrization, and further reduced and stabilized by the running of the coupling.

The relative importance of various contributions to the resummation is elucidated by comparing in Fig. 8 the slope of the resummed running-coupling splitting function (Fig. 17) with respect to $\xi$ to that of the NLO GLAP result, and that of the splitting function obtained by inverse Mellin transform of the Bateman anomalous dimension $\gamma^{B}\left(\alpha_{s}(t), N\right)$ in Eq. (7). It is clear that for $x \gtrsim 0.1$ the slope (and thus, up to a constant, the splitting function) is determined by the GLAP result, and for $x \lesssim 10^{-2}$ by the Bateman result [25]. Note that this is despite the fact that it is only for tiny values of $x \lesssim 10^{-15}$ that the Bateman asymptotic slope reduces (to within about $10 \%$ ) to its asymptotic value $N_{0}$ (the location of the leading singularity) 25].

Hence the qualitative features of the resummation are essentially determined by matching to the GLAP result the Bateman anomalous dimension, which in turn is fully determined by resummation of the running coupling terms Eq. (5), and thus by the intercept and shape of the minimum of the symmetrized kernel displayed in Fig. 5 [10. The insensitivity of these to the details of the resummation procedure explains the stability of the results. The dominant feature of the result in the HERA region $10^{-2} \gtrsim x \gtrsim 10^{-4}$ is a dip in the splitting function which results from this matching (see Ref. [16] for an alternative discussion of this feature of resummed results).

A resummed gluon splitting function was also presented in Refs. [19|20]. The agreement is reasonable at the qualitative level, but the resummed splitting function appears to display a stronger rise at small $x$ and a somewhat more pronounced dip at intermediate $x$. This may be due to the fact that in Ref. [19/20] no symmetrization of the kernel is performed: this, as discussed above, leads to resummed results which tend to be unstable upon perturbative corrections as $x \rightarrow 0$.

\section{Quarks and phenomenology}

Going from a resummation of the evolution equations at $N_{f}=0$ to fully resummed physical observables requires two ingredients: the inclusion of the quark sector in the resummation, and resummed partonic cross sections.

\subsection{The resummed splitting function ma- trix}

An extension of small $x$ BFKL-like evolution equations to the quark sector has been attempted in Ref. [18, but results in a fully consistent factorization scheme are still not available. However, it turns out that this construction is not necessary for the determination of the matrix of resummed splitting functions. This is due to the fact that the resummation only affects one of the two eigenvectors of the singlet anomalous dimension matrix. Therefore, in order to obtain coupled resummed evolution equations for singlet quarks and gluons it is sufficient to fix the factorization scheme at the resummed level [17.

Indeed, calling $\gamma^{+}$and $\gamma^{-}$the two eigenvectors of the anomalous dimension matrix, if only contributions to $\gamma$ which are singular as $N \rightarrow 0$ are included, $\gamma^{-}=0$ and thus

$$
\begin{gathered}
\gamma^{g g}+\gamma^{q q}=\gamma^{+} \\
\gamma^{g g} \gamma^{q q}=\gamma^{q g} \gamma^{g q} .
\end{gathered}
$$

up to nonsingular terms. Furthermore, in the $\overline{\mathrm{MS}}$ and related schemes, $\gamma^{g q}=\frac{C_{F}}{C_{A}} \gamma^{g g}$ (up to nonsingular terms) 27. Hence, combining the determination of $\gamma^{+}$with the knowledge of $\gamma^{q g}$ in the $\overline{\mathrm{MS}}$ scheme, which was computed at the resummed level in Ref. 27, the anomalous dimension matrix is fully determined.

The actual computation of the full splitting function matrix with $n_{f} \neq 0$ entails some technical complications, which have only recently been solved in Ref. [17. Firstly, when $n_{f} \neq 0$, the eigenvectors of the anomalous dimension matrix develop an unphysical singularity at the value of $N$ where the two eigenvectors cross. This singularity cancels in the solution to evolution equations, and the cancellation must be enforced ex- 

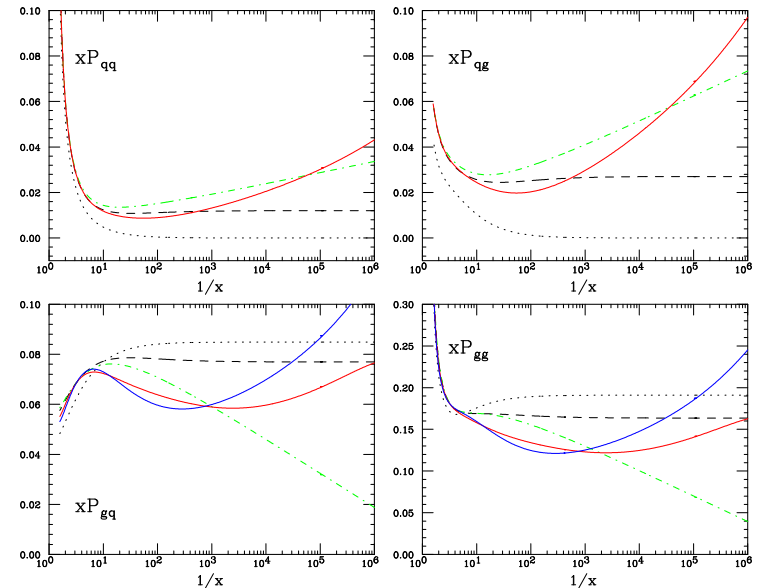

Figure 9. The splitting function matrix with $n_{f}=4, \alpha_{s}=0.2$. The curves correspond to LO (dotted), NLO (dashed), NNLO (dot-dash), resummed (solid). The two different resummed curves (in the gluon sector) correspond to the $\overline{\mathrm{MS}}$ (steeper at small $x$ ) and $\mathrm{Q}_{0} \overline{\mathrm{MS}}$ factorization schemes.

actly throughout the double-leading resummation and symmetrization: if it were spoiled by subleading terms, this would lead to a spurious small $x$ rise of parton distributions. Furthermore, the quark-sector anomalous dimension $\gamma_{q g}$ was determined in Ref. 27] in the $\overline{\mathrm{MS}}$ scheme, while the double-leading resummation is most naturally performed in the $\mathrm{Q}_{0}$ scheme 2928: in the $\overline{\mathrm{MS}}$ scheme the running coupling terms Eq. (5) are factored order by order in the coefficient function. Because their resummation determines the leading small $x$ behaviour, as discussed in Sect. 2.3, it is more convenient to perform the resummation in a scheme where they are included in the splitting function. Whereas the scheme change from the $\mathrm{Q}_{0}$ scheme to $\overline{\mathrm{MS}}$ in the pure-gluon case was worked out previously [29|28], its construction in the presence of quarks is nontrivial 2217].

The resummed matrix of splitting functions in the $\overline{\mathrm{MS}}$ and $\mathrm{Q}_{0} \overline{\mathrm{MS}}$ factorization schemes is compared to the unresummed result in Fig. 9, Whereas a detailed comparison with the CCSS splitting function matrix 18 is not possible, because CCSS results are given in a scheme which differs from the standard $\overline{\mathrm{MS}}$ scheme by an amount which is undetermined beyond fixed NLO, a qualitative comparison shows reasonable agreement.

Quark sector splitting functions have also been given in Ref. 20] (see also Ref. 21]). Their agreement with those of Fig. 9 is not so good: the $P_{q g}$ splitting function shows a much stronger small $x$ rise, and a sizable deviation from the NLO result at large $x \gtrsim 0.1$. The latter feature can be understood as a consequence of the fact that in Refs. 19|20 no matching to large $x$ is performed: contributions from the small $x$ resummation in the large $x$ region are not subtracted. The former feature is likely to be due to the fact that results in Ref. 20] are not determined in a fully consistent factorization scheme. In fact, the resummation is performed in the $\mathrm{Q}_{0}$ scheme, but it is then combined with $\overline{\mathrm{MS}}$ (or DIS) scheme coefficient functions: in the TW approach, the issue of the scheme transformation from $\mathrm{Q}_{0}$ to $\overline{\mathrm{MS}}$ is still unresolved. Because of the aforementioned interplay between the scheme choice and the resummation of running coupling singularity, this inconsistency is likely to affect strongly the small $x$ behaviour.

\subsection{Coefficient function resummation}

The leading small $x$ contributions to partonic cross sections are known to all orders for a small but increasing number of physical processes: they were first computed for heavy quark photo-and electroproduction in Ref. 31] (later extended to hadroproduction in Ref. [32]), they have been determined for deep-inelastic scattering in Ref. [27] and more recently for Higgs production 33 and the Drell-Yan processes 34 .

Expressions for coefficient functions in the NLO of the double-leading expansion were already constructed in Ref. 7], where, however, the running coupling terms discussed in Sect. 2.3 were still left unresummed, thereby simplifying issues of scheme dependence, but at the cost of not reproducing the correct small $x$ behaviour. However, running coupling corrections to the resummation of coefficient functions also grow as $\xi \rightarrow$ $\infty$, analogously to the running coupling corrections to splitting functions Eq. 55 they must be resummed lest physical observables develop un- 

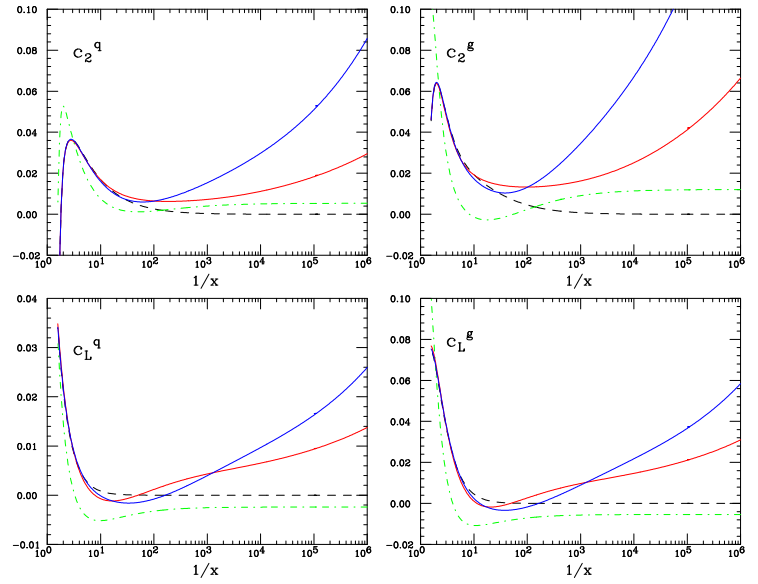

Figure 10. The matrix of coefficient functions with $n_{f}=4, \alpha_{s}=0.2$. The curves correspond to NLO (dashed), NNLO (dot-dash), resummed (solid). The two different resummed curves (in the gluon sector) correspond to the $\overline{\mathrm{MS}}$ (steeper at small $x$ ) and $\mathrm{Q}_{0} \overline{\mathrm{MS}}$ factorization schemes.

physical singularities which leads to a spurious small $x$ growth 30 .

The resummation can be performed exactly 30] when the double-leading expression of the coefficient function is known in closed form. This is however not the case for the $F_{2}$ deepinelastic coefficient function in the $\overline{\mathrm{MS}}$ scheme, for which only a series expansion generated by a recursion relation is available [27]. In such case, the dominant running coupling corrections to the coefficient function can be resummed through a divergent asymptotic expansion, which may be summed by the Borel method [17. The ensuing resummed coefficient functions are displayed in Fig. 10. Resummed coefficient functions were also presented in Ref. 20] (also including running coupling resummation) and are qualitatively similar, though a detailed comparison is hampered by the fact that the factorization scheme used there is different (DIS instead of $\overline{\mathrm{MS}}$ ).

\subsection{Parton distributions and structure functions}

Combining the ingredients discussed so far it is possible to determine resummed predictions for deep-inelastic structure functions. Eventually, these should be used, together with resummed
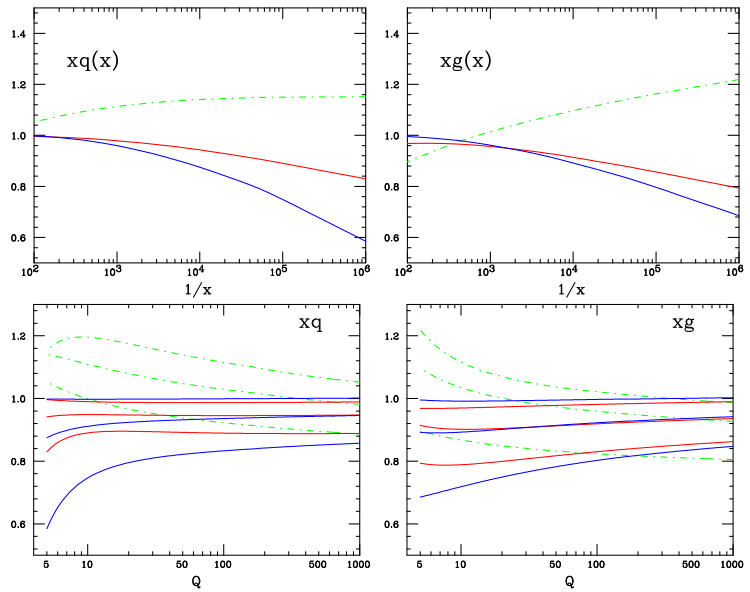

Figure 11. The ratio of the resummed (solid) or NNLO (dot-dashed) to NLO singlet quark and gluon distributions as a function of $x$ at the scale $Q_{0}=5 \mathrm{GeV}$ (top) and as a function of $Q$ at fixed $x=10^{-2}, 10^{-4}, x=10^{-6}$ (bottom; the smallest $x$ is the lowest curve in the resummed case and the highest at NNLO). The ratios are determined assuming that the structure functions $F_{2}$ and $F_{L}$ are kept fixed at the scale $Q_{0}=5 \mathrm{GeV}$. The two different resummed curves correspond to the $\overline{\mathrm{MS}}$ (smaller at small $x$ ) and $\mathrm{Q}_{0} \overline{\mathrm{MS}}$ factorization schemes.

expressions for other physical processes, for the determination of parton distributions at the resummed level.

An estimate of the impact of resummation on parton distributions can be obtained by first computing the structure functions $F_{2}$ and $F_{L}$ with some typical "toy" set of NLO parton distributions (PDFs), and then assuming that the structure functions are kept fixed at some scale: this is then enough to determine the resummed singlet quark and gluon distribution at that scale. The effect on PDFs is close to that which would be obtained if PDFs were determined from a fit to DIS data mostly clustered around that scale. Results for the typical HERA (compare Fig. 1) scale choice of $\mathrm{Q}^{2}=25 \mathrm{GeV}^{2}$ are shown in Fig. 11. where we display the singlet quark and gluon distributions as a function of $x$ at this starting scale, and then as a function of $Q^{2}$ for various $x$ values. Results are shown as a ratio of the resummed or 
NNLO result to the NLO one. The impact of the resummation at the "HERA scale" is comparable to that of NNLO corrections, but it goes in the opposite direction: it tends to suppress the starting PDFs while the NNLO tends to enhance them. When evolving up the differences tend to be washed out because of asymptotic freedom.

The PDFs displayed in Fig. 11 can then be used to determine $K$-factors for resummed structure functions: these were computed in Ref. [17. There the factorization and renormalization scale dependence of the of results were also studied, and shown to be small. Indeed, once all ingredients are included, the resummed perturbative expansion of physical observables turns out to be as good as the standard fixed-order expansion.

The dominant qualitative feature of the $K_{-}^{-}$ factors is that resummation leads to a suppression of the structure functions $F_{2}$ and $F_{L}$ at small $x$. The $K$-factors were compared to those determined using the TW approach [20] in Ref. 21]. The main differences are related to the fact that TW $K$-factors can differ sizably from one even at $x \gtrsim 10^{-2}$ where the ABF result matches smoothly to the NLO one, and that the TW $K$ factors still show a marked scale dependence at large $Q^{2} \gtrsim 10^{4} \mathrm{GeV}^{2}$ where the scale dependence of the ABF result flattens out completely. These differences are likely to be due to the various features of the TW approach mentioned above: in particular, the lack of matching terms to large $x$, and the fact that the factorization scheme is not treated in a fully consistent way. Indeed, there appears [17 to be a significant cancellation of scheme dependence between evolved parton distributions and coefficient functions, which is inevitable spoiled if these are not determined in the same factorization scheme.

$K$-factors can also be used to determine resummed predictions in an approximate way, by first, computing the $K$ factors as discussed above, but using a realistic underlying set of PDFs, then verifying that the values of the $K$ factors are reasonably independent of the choice of underlying PDFs, and finally using the $K$-factors to correct NLO predictions obtained from any PDF set.

In Fig. 12 we show the structure functions $F_{2}$ and $F_{L}$ at the scale $Q^{2}=M_{W}^{2}$ obtained applying
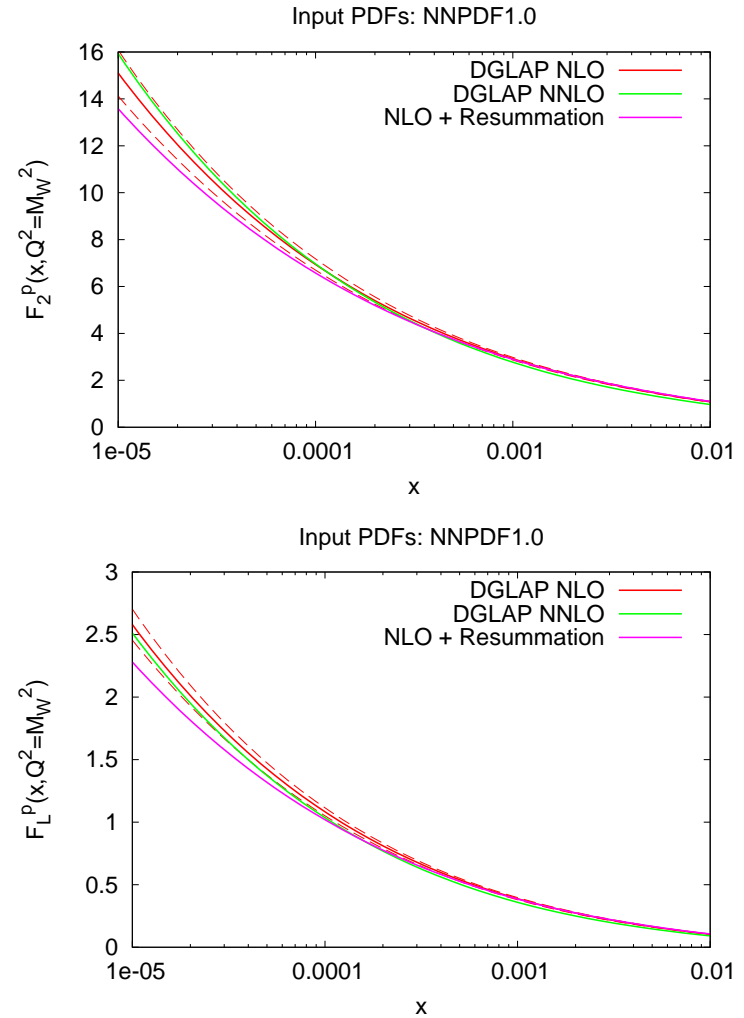

Figure 12. The deep-inelastic structure functions $F_{2}$ and $F_{L}$ at $Q^{2}=M_{W}^{2}$, computed at NLO, NNLO and resummed level. The parton distributions are from the NLO NNPDF1.0 set in the NLO case, while at the NNLO and resummed level they are computed using $K$-factors as discussed in the text. The PDF uncertainty band is shown for the NLO prediction, while only the central prediction is displayed in the other cases.

either resummed or NNLO $K$-factors to the NLO prediction from the NNPDF1.0 parton set 35. Interestingly, the suppression due to the resummation in the small $x \lesssim 10^{-4}$ region is larger than the one-sigma band due to PDF uncertainties.

These results show that resummation is relevant for deep-inelastic scattering at a highenergy hadron-electron collider such as a collider based on the LHC (LHeC [36]). It will be interesting to study the impact on precision LHC processes such as $\mathrm{W}$ and $\mathrm{Z}$ production of resummation corrections, both from their direct effect on 
partonic cross sections, and their indirect effect due to their impact on the extraction of parton distributions.

In summary, we have seen that the impact of resummation is as large as that of NNLO corrections in the HERA region. The theoretical framework on which resummed results are based is now on a similar footing as that of standard fixed order perturbation theory, whose reliability is thus extended also to kinematical regions, relevant for HERA and the LHC, where NNLO results become unstable.

Acknowledgements: We thank M. Ciafaloni, D. Colferai, G. Salam, A. Stasto, R. Thorne and C. White for various discussions, and J. Rojo for help with the NNPDF prediction of Fig. 12. This work was partly supported by the Scottish Universities' Physics Alliance, by the Italian PRIN program for 2007-08, and by the Marie Curie Research and Training network HEPTOOLS under contract MRTN-CT-2006-035505.

\section{REFERENCES}

1. M. Dittmar et al., arXiv:hep-ph/0511119.

2. R. S. Thorne, arXiv:0808.1845 [hep-ph].

3. R. D. Ball and S. Forte, Phys. Lett. B 335 (1994) 77.

4. F. Caola and S. Forte, Phys. Rev. Lett. 101 (2008) 022001.

5. R. D. Ball and S. Forte, Phys. Lett. B465 (1999) 271.

6. G. Altarelli, R. D. Ball and S. Forte, Nucl. Phys. B575, 313 (2000);

7. G. Altarelli, R. D. Ball and S. Forte, Nucl. Phys. B599 (2001) 383;

8. G. Altarelli, R. D. Ball and S. Forte, Nucl. Phys. B 621 (2002) 359.

9. G. Altarelli, R. D. Ball and S. Forte, Nucl. Phys. B 674 (2003) 459;

10. G. Altarelli, R. D. Ball and S. Forte, Nucl. Phys. B 742 (2006) 1,

11. G. Salam, JHEP9807 (1998) 19.

12. M. Ciafaloni and D. Colferai, Phys. Lett. B452 (1999) 372.

13. M. Ciafaloni, D. Colferai and G. P. Salam, Phys. Rev. D 60 (1999) 114036.

14. M. Ciafaloni, D. Colferai and G. P. Salam, JHEP 0007 (2000) 054
15. M. Ciafaloni, D. Colferai, G. P. Salam and A. M. Stasto, Phys. Rev. D 66 (2002) 054014; Phys. Lett. B 576 (2003) 143; Phys. Rev. D 68 (2003) 114003.

16. M. Ciafaloni, D. Colferai, G. P. Salam and A. M. Stasto, Phys. Lett. B 587 (2004) 87.

17. G. Altarelli, R. D. Ball and S. Forte, Nucl. Phys. B 799 (2008) 199.

18. M. Ciafaloni, D. Colferai, G. P. Salam and A. M. Stasto, JHEP 0708 (2007) 046.

19. R. S. Thorne, Phys. Rev. D 60 (1999) 054031; Phys. Lett. B 474 (2000) 372; Phys. Rev. D 64 (2001) 074005.

20. C. D. White and R. S. Thorne, Phys. Rev. D 75 (2007) 034005.

21. Proceedings of the 2008 HERA-LHC workshop, to be published.

22. R. D. Ball and S. Forte, Phys. Lett. B 351 (1995) 313

23. T. Jaroszewicz, Phys. Lett. B 116 (1982) 291.

24. R. D. Ball and S. Forte, Nucl. Phys. B 742 (2006) 158

25. C. Frugiuele, Masters Thesis, Milan University (2007).

26. R. D. Ball and S. Forte, Phys. Lett. B 359 (1995) 362.

27. S. Catani and F. Hautmann, Phys. Lett. B315 (1993) 157; Nucl. Phys. B427 (1994) 475 .

28. M. Ciafaloni and D. Colferai, JHEP 0509 (2005) 069;

S. Marzani, R. D. Ball, P. Falgari and S. Forte, Nucl. Phys. B 783 (2007) 143

29. M. Ciafaloni, Phys. Lett. B356 (1995) 74.

30. R. D. Ball, Nucl. Phys. B 796 (2008) 137.

31. S. Catani, M. Ciafaloni and F. Hautmann, Nucl. Phys. B 366 (1991) 135.

32. R. D. Ball and R. K. Ellis, Jour. High Energy Phys. 0105 (2001) 053.

33. S. Marzani, R. D. Ball, V. Del Duca, S. Forte and A. Vicini, Nucl. Phys. B 800 (2008) 127; arXiv:0809.4934 [hep-ph].

34. S. Marzani and R. D. Ball, arXiv:0812.3602 [hep-ph].

35. R. D. Ball et al. [NNPDF Collaboration], Nucl. Phys. B 809 (2009) 1.

36. M. Klein et al., Proceedings of 11th European Particle Accelerator Conference (EPAC 08). 